\title{
TRATAMENTO DA DOENÇA DE CROHN COM INFLIXIMABE: PRIMEIRA OPÇÃO?
}

\section{Treatment of Crohn's disease with infliximab: first choice?}

\author{
Anna Paula Rocha MALHEIROS, Magaly Gemio TEIXEIRA, Arceu SCANAVINI NETO, \\ Edésio Vieira SILVA FILHO, Leonardo Correa de Oliveira RODRIGUES, \\ Roberta THIERRY, Sergio NAHAS, Ivan CECCONELLO
}

\begin{abstract}
Malheiros APR, Teixeira MG, Scanavini Neto A, Silva Filho EV, Rodrigues LCO, Thierry R, Nahas S, Cecconello I. Tratamento da doença de Crohn com infliximabe: primeira opção? ABCD Arq Bras Cir Dig 2009;22(2):101-4

RESUMO - Racional - A doença de Crohn é uma inflamação crônica do trato gastrointestinal ainda de difícil tratamento. Há busca permanente de novos agentes que possam colaborar com a melhoria dos resultados. O infliximabe é um anticorpo monoclonal quimérico anti-fator de necrose tumoral alfa e está indicado na doença de Crohn refratária e fistulizante. Objetivo - Observar os resultados do tratamento da doença de Crohn com o anti-fator de necrose tumoral alfa. Métodos - Estudo prospectivo de 60 doentes com doença de Crohn no período de seis anos. Foram considerados como critérios de exclusão: infecção atual ou nos últimos três meses; diagnóstico de tuberculose; quadro clínico e/ou radiológico de oclusão intestinal parcial ou total; gravidez ou amamentação. Todos os doentes foram previamente submetidos à radiografia do tórax, leucograma e intradermo reação para tuberculose. Foram tratados com infliximabe na dose de $5 \mathrm{mg} / \mathrm{kg}$ de peso, aplicado por via endovenosa a intervalos de dois meses. Os doentes foram divididos em três grupos de acordo com o tempo de doença, isto é, grupo 1 aqueles com até cinco anos de diagnóstico, grupo 2 com seis até 10 anos e grupo 3 com mais de 10 anos de diagnóstico. Os resultados foram considerados subjetivamente através de protocolo após cada aplicação como: melhor, pior ou inalterado em relação ao estado geral do doente, sintomas intestinais e doença perianal. Resultados - No tratamento inicial $76 \%$ dos pacientes responderam ao anticorpo. Observou-se que após a primeira dose da medicação, os com mais de 10 anos de doença e submetidos à operação abdominal tiveram resultado satisfatório semelhantemente aqueles doentes com menos de cinco anos de doença e não operados (62,5\% $\mathrm{e}$ $80 \%$ respectivamente dos doentes que melhoraram), sendo este resultado estatisticamente significativo. Conclusão - O tratamento com infliximabe mostrou-se eficaz e tolerável no manejo dos sintomas dos pacientes com doença de Crohn ativa que não respondem ao tratamento convencional.

DESCRITORES - Doença de Chohn. Doença inflamatória intestinal. Terapia monoclonal.
\end{abstract}

\section{INTRODUÇÃO}

O tratamento convencional da doença de Crohn (DC) é muitas vezes desapontador. Apesar da variedade de drogas disponíveis para o seu tratamento, tais como: salicilatos e seus derivados, corticosteróides, antibióticos e imunossupressores, nenhuma destas mostrou ser totalmente eficaz ou definitiva para o tratamento da doença e seus surtos de exacerbação. As drogas biológicas foram desenvolvidas com o objetivo de serem mais específicas para esse tipo de tratamento.

Sabe-se que o fator de necrose tumoral (TNF) alfa está elevado nas fezes, mucosa e sangue dos doentes com DC, e que o desequilíbrio entre secreção e sua inibição está relacionado com a patogênese da doença ${ }^{13}$. Neste contexto, algumas drogas foram desenvolvidas com a estratégia de bloqueá-lo e, demonstraram potente atividade clínica, confirmando o seu papel na patogênese da DC e no tratamento de doentes de difícil manejo clínico ${ }^{5,15,17}$.

Entre os variados tipos de anti-TNF alfa que surgiram

Trabalho realizado na Disciplina de Coloproctologia do Departamento de Gastroenterologia da Faculdade de Medicina da Universidade de São Paulo, São Paulo, SP, Brasil.

Endereço para correspondência: Anna Paula Rocha Malheiros, e-mail: ap-malheiros@bol.com.br para o tratamento da DC podemos citar: infliximabe, adalimumabe, CDP870, CDP571, etanercept e ornecept ${ }^{17}$.

O infliximabe é um anticorpo monoclonal IgG1 quimérico constituído de $75 \%$ de proteína humana e $25 \%$ de proteína de camundongo. A porção de camundongo contém o sítio de ligação para o fator de necrose tumoral (TNF) alfa, enquanto a porção humana é responsável pela função efetora. O infliximabe liga-se ao TNF alfa solúvel e TNF ligado à membrana bloqueando as atividades biológicas da citocina ${ }^{2,3}$.

O presente estudo visa avaliar os resultados obtidos com o uso do infliximabe no tratamento dos doentes com DC e observar se houve diferença na resposta clínica entre os grupos de doentes que variaram quanto ao tempo de doença e tratamento cirúrgico prévio relacionado.

\section{MÉTODO}

O estudo foi prospectivo observando-se 60 doentes com DC atendidos no ambulatório de doenças inflamatórias do Serviço de Cirurgia do Cólon e Reto da Divisão de Clínica Cirúrgica II do Hospital das Clínicas da Faculdade de Medicina da Universidade de São Paulo (HCFMUSP), no período de julho de 1999 a dezembro de 2005. 
O projeto de pesquisa foi aprovado pelo comitê de ética desta instituição e obteve-se consentimento informado por escrito de todos os pacientes.

Foram considerados como critérios de exclusão: infecção atual ou nos últimos três meses; diagnóstico de tuberculose; quadro clínico e/ou radiológico de oclusão intestinal parcial ou total; gravidez ou amamentação. Todos os doentes foram previamente submetidos à radiografia do tórax, leucograma e intradermo reação para tuberculose.

Os doentes foram tratados com infliximabe na dose de $5 \mathrm{mg} / \mathrm{kg}$ de peso, aplicado por via endovenosa a intervalos de dois meses, no Hospital Dia do HCFMUSP em período de, no mínimo, duas horas de infusão, sob supervisão de um profissional médico do próprio Hospital Dia. As medicações de uso oral e diário dos doentes foram mantidas durante o tratamento com o anti-TNF alfa de acordo com as orientações do médico responsável.

$\mathrm{Na}$ análise dos dados, os doentes foram divididos em três grupos de acordo com o tempo de doença, isto é, grupo 1 aqueles com até cinco anos de diagnóstico de DC, grupo 2, com seis até dez anos de diagnóstico de DC, e grupo $3 \mathrm{com}$ mais de dez anos de diagnóstico de DC. Os resultados foram considerados subjetivamente através de protocolo após cada aplicação como: melhor, pior ou inalterado em relação ao estado geral do doente, sintomas intestinais e doença perianal.

Para análise estatística o teste de Mann-Whitney foi usado para comparar as variáveis numéricas: idade, número de doses, tempo de sintomatologia e diagnóstico, tempo de doença, intervalo de tempo entre as doses, em relação aos grupos: primeira dose até a dose 14, e o teste Gama para comparação dos resultados entre as faixas etárias.

Para todos os testes foi utilizado um alfa de $5 \%$, sendo estatisticamente significantes os testes com $P<0,05$. O software utilizado para a realização dos cálculos foi o SPSS $^{\circledR 9} 9.0$ for Windows ${ }^{\mathrm{TM}}$.

\section{RESULTADOS}

Avaliou-se 60 doentes com DC conforme características demonstradas na Tabela 1.

TABELA 1 - Distribuição dos doentes com DC segundo características principais

\begin{tabular}{ll}
\hline Homens & 27 \\
Mulheres & 33 \\
\hline Idade mediana (anos) & $34(14$ a 60$)$ \\
\hline Localização da DC (ID=intestino delgado, IG=intestino & $\mathbf{N}(\%)$ \\
grosso e PA=perianal) & \\
ID + IG +PA & $25(41,7 \%)$ \\
ID + PA & $15(25 \%)$ \\
IG + PA & $14(23,3 \%)$ \\
ID & $3(5 \%)$ \\
ID + IG & $2(3,3 \%)$ \\
IG & $1(1,7 \%)$ \\
\hline Doentes em uso de outras drogas concomitantemente & $40(66,7 \%)$ \\
Doentes em uso de prednisona & $25(41,7 \%)$ \\
\hline Tempo mediano de diagnóstico da DC (anos) & $7(1 \mathrm{a} 28)$ \\
\hline Operação abdominal anterior & $34(56,7 \%)$ \\
Operação orificial anterior & $38(63,3 \%)$ \\
\hline Número total aplicações de anti-TNF alfa & 225 \\
Média de doses aplicadas de anti-TNF alfa & $3,7(1 \mathrm{a} 14)$ \\
\hline
\end{tabular}

Nenhum dos pacientes estudados apresentou doença perianal isoladamente como manifestação da DC.

Observou-se que, em relação aos resultados obtidos após a primeira dose da medicação, os doentes com mais de dez anos de doença e submetidos à operação abdominal tiveram resultado satisfatório semelhantes aqueles com menos de cinco anos de doença e não operados (Gráficos 1 e 2), sendo este resultado estatisticamente significativo $(P<0,05)$.

\section{MELHORA CLÍNICA}

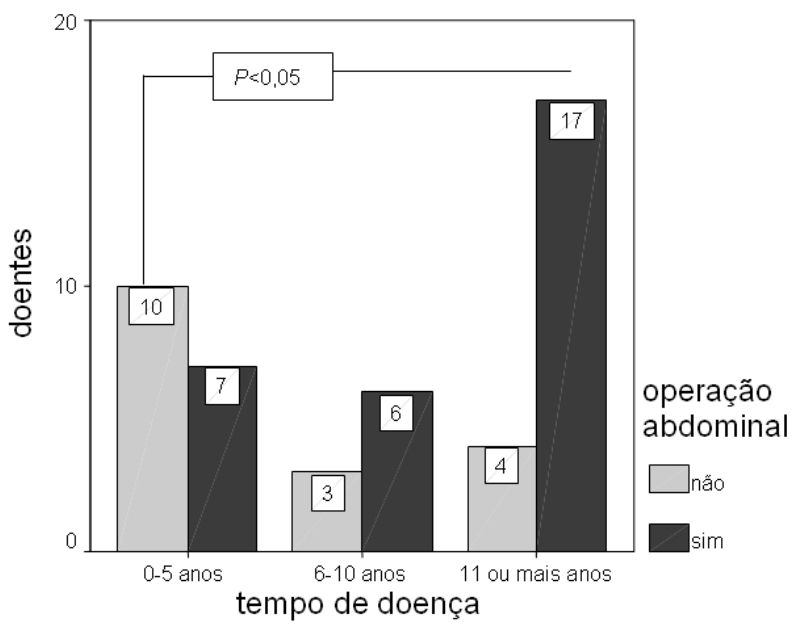

GRÁFICO 1 - Distribuição dos doentes com DC segundo a melhora clínica com o tratamento com anti-TNF alfa em relação ao tempo de diagnóstico e história prévia de operação abdominal

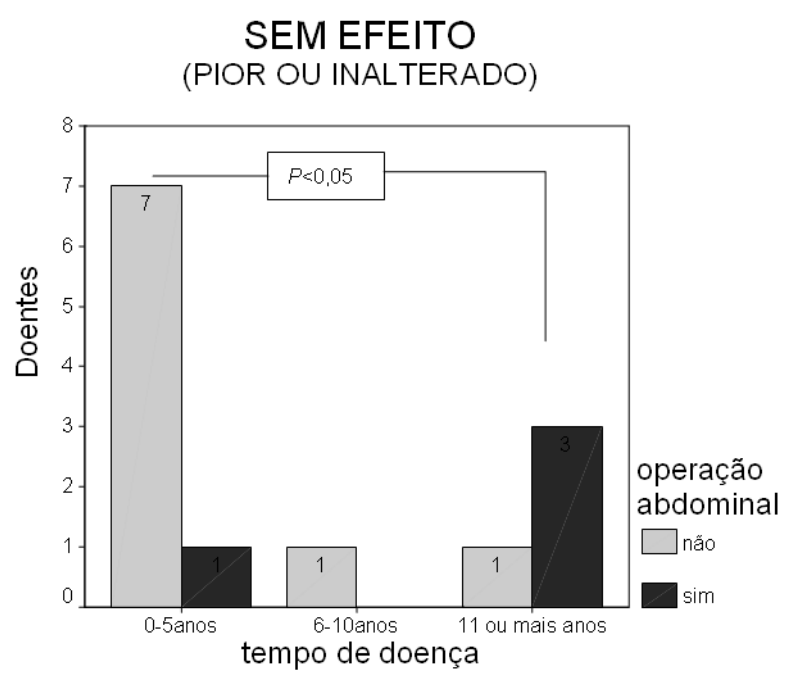

GRÁFICO 2 - Distribuição dos doentes com DC segundo a piora clínica ou resultado inalterado com o tratamento com anti-TNF alfa em relação ao tempo de diagnóstico e história prévia de operação abdominal

A principal indicação para o emprego do anti-TNF alfa foi a presença de doença perianal em 36 casos $(60 \%)$ e a intratabilidade clínica em 24 casos (40\%).

A presença de efeitos colaterais graves ocorreu em 
cinco casos, sendo três de leucopenia que necessitaram de interrupção do tratamento; um caso de oclusão intestinal em que o doente foi operado de urgência; e um caso de pneumonia em um doente com quadro grave de DC em que o uso do infliximabe foi indicado como última opção de tratamento pela gravidade do quadro clínico.

\section{DISCUSSÃO}

A incidência da DC é relativamente incomum, variando de 0,08 a 7/1.000.000 habitantes na Europa ${ }^{1}$. Esses valores são variáveis de acordo com a localização geográfica, fatores socioeconômicos e ambientais. Os objetivos do tratamento são: a remissão por longo prazo, diminuição do número e período de internações hospitalares e operações.

A falta de resultados mais efetivos com as drogas habitualmente utilizadas (salicilatos e seus derivados, antibióticos, corticosteróides e imunossupressores) estimulou o desenvolvimento de novos tratamentos com o objetivo de melhorar a qualidade de vida destes doentes. Sendo o principal avanço alcançado: a introdução das drogas biológicas.

Van Dullemen et al. ${ }^{18}$ sugeriram (1995) que a chave no tratamento da DC seria o bloqueio do TNF alfa. Targan et al. ${ }^{17}$ descreveram a eficácia do uso do anti-TNF como estratégia no tratamento da DC.

Lichetenstein et al. ${ }^{12}$ descreveram os benefícios do anti-TNF alfa, principalmente na DC, como modulador do tratamento cirúrgico, demonstrando diminuição do número de hospitalizações e procedimentos operatórios de urgência ${ }^{12}$.

$\mathrm{O}$ inflixmabe foi o primeiro anti-TNF alfa introduzido para uso na prática clínica nos Estados Unidos em 1998 ${ }^{11}$. No Brasil, essa mesma medicação foi incluída pela Secretaria de Saúde Pública no tratamento da DC no ano de 2002. Os resultados favoráveis com o uso dela levou estes autores a estudar seus resultados nos doentes tratados.

O intervalo de tempo de diagnóstico da doença não influenciou os resultados satisfatórios com o uso do anticorpo na primeira dose. No entanto, eles foram mais expressivos tanto no grupo com menos de cinco anos quanto no grupo com mais de dez anos de tempo de doença.

As principais indicações descritas na literatura para o uso do infliximabe na DC são: DC moderada a grave refratária ao tratamento convencional apesar da terapia adequada com corticosteróides e/ou imunossupressores (AZA, 6-MP ou metotrexate), e DC fistulizante em pacientes que não responderam adequadamente ao tratamento convencional, incluindo, antibióticos e drenagem cirúrgica sob anestesia e imunossupressores ${ }^{9,10}$. Neste grupo as indicações mais frequentes foram a doença perianal e intratabilidade.

O ACCENT I foi o maior e mais importante estudo multicêntrico randomizado envolvendo o infliximabe publicado em 2002. O estudo fortaleceu a indicação do tratamento após demonstrar seus benefícios em 573 doentes de 45 centros envolvendo: América do Norte, Europa e Israel. Foram randomizados três grupos de doentes que utilizaram doses diferentes da droga: grupo 1 que recebeu dose única do anticorpo $(5 \mathrm{mg} / \mathrm{kg} / \mathrm{peso})$, o grupo 2 que recebeu três doses de $5 \mathrm{mg} / \mathrm{kg} /$ peso no intervalo de 0,2 e 6 semanas e doses de manutenção de $5 \mathrm{mg} / \mathrm{kg} /$ peso a cada oito semanas até 46 semanas, e o grupo 3 que recebeu as mesmas três doses de indução do grupo anterior e manutenção com doses de $10 \mathrm{mg} / \mathrm{kg} /$ peso a cada oito semanas até completar 46 semanas. Todos foram avaliados após o período de 54 semanas, e observou-se remissão da doença em 14\% do primeiro grupo, em $29 \%$ do segundo grupo e $39 \%$ do terceiro grupo, houve melhora clínica em 16\%, 38\% e 47\% respectivamente neste mesmo período ${ }^{6}$.

O ACCENT II foi idealizado para demonstrar o impacto da terapia de manutenção a cada oito semanas, nos pacientes com doença fistulizante e refratária. Neste, os autores observaram que a terapia de indução com doses nas semanas 0,2 e 6 associada a manutenção do uso da droga a cada dois meses proporcionou a remissão clínica das fistulas em $46 \%$ dos casos após 54 semanas em comparação com $23 \%$ daqueles que não receberam a terapia de manutenção ${ }^{16}$.

A abordagem utilizada pelos autores deste trabalho neste grupo inicial de 60 doentes, revelou que a terapia de doses a cada oito semanas mostrou índices de sucesso no tratamento semelhantes aos estudos já publicados, quando esta casuística é avaliada até a quinta dose. Observou-se que em todos os três aspectos (estado geral, sintomas intestinais e doença perianal) houve melhora clínica superior a $60 \%$.

Estes índices de sucesso avaliados estatisticamente até o período de 32 semanas, com intervalos de oito semanas entre as doses, sem a aplicação das doses de indução $(0,2$ e 6 semanas) foram semelhantes aos obtidos com os estudos multicêntricos apresentados ${ }^{6,16}$.

Em 26 centros da Europa, foi realizado um estudo multicêntrico randomizado o qual demonstrou que o uso da terapia primária com o anti-TNF alfa em pacientes recém diagnosticados ou com doença ativa há menos de quatro anos causou menor taxa de recidiva da DC, redução do uso de corticosteróide e período de ausência de sintomatologia significativamente maior quando comparado ao grupo que utilizou a terapia convencional. Os autores também relataram o desaparecimento de sinais de ulceração da mucosa em $73 \%$ dos doentes tratados com o anticorpo contra $30 \%$ dos que não utilizaram a terapia biológica ${ }^{8}$.

Neste estudo, verificou-se que as melhores respostas ao uso de terapia biológica ocorreram no grupo com pouco tempo de duração da doença nos quais ainda não haviam se instalado as complicações (por exemplo, estenose), ou no grupo com mais de 10 anos de evolução. Este grupo era constituído em sua maioria por doentes já operados e, portanto, sem complicações da doença, ou seja, estavam se comportando como doentes em fase inicial da doença. Estes resultados corroboram a idéia de que o uso da terapia biológica como droga de escolha inicial seja benéfica para os pacientes.

As divergências quanto ao uso precoce da droga biológica no tratamento precoce da DC inclui desde custos, efetividade, riscos e efeitos colaterais. Porém alguns autores como Salborn et al. ${ }^{15}$ e D'Haens et al. ${ }^{4}$ que discutem frequentemente sobre este tipo de estratégia de tratamento denominada de "top-down" ou inversão da pirâmide de tratamento, afirmam que a ação mais agressiva no início da doença poderia prevenir a sua progressão e possivelmente 
suas complicações ${ }^{6,14}$.

Apesar dos efeitos colaterais passíveis de serem observados pela imunogenicidade da droga em especial o infliximabe, alguns dos novos agentes como o adalimumabe e certolizumabe, por não conterem a porção murínica na sua composição parecem oferecer menor risco de respostas alérgicas relacionadas à infusão e auto-imunidade ${ }^{7,11}$.

As drogas biológicas podem também oferecer estabilidade mais precoce da doença, com diminuição do risco de complicações como fístula ou estenoses, e provavelmente alterar o seu curso natural associado ao número de internações e operações de urgência. Seu uso é advogado inclusive no tratamento pós-operatório de ressecções ileocecais para prevenção de recorrência em casos graves ${ }^{17}$.

Esse tipo de abordagem não é consenso na prática clínica. Mais estudos são necessários para que se possa fundamentar tal medida terapêutica.

\section{CONCLUSÕES}

O tratamento com anti-fator de necrose tumoral alfa, obedecidas as indicações precisas, associou-se a baixo índice de efeitos colaterais graves tendo apresentado bons resultados na resolução da doença de Crohn perianal, na melhora da sintomatologia intestinal e no estado geral dos pacientes.

Malheiros APR, Teixeira MG, Scanavini Neto A, Silva Filho EV, Rodrigues LCO, Thierry R, Nahas S, Cecconello I. Treatment of Crohn's disease with in infliximab: first choice?ABCD Arq Bras Cir Dig 2009;22(2):101-4

ABSTRACT - Background - Crohn's disease is a chronic inflammatory disorder of the gastrointestinal tract with difficult management. Infliximab is a chimeric IgG1 monoclonal antibody against tumor necrosis factor and is indicated for refractory luminal and fistulization in Crohn's disease. Aim - To observe the outcome of 60 patients with diagnosis of Crohn's disease treated with infliximab. Methods - Prospective study with 60 patients with Crohn's disease in six years of observation. Exclusion criteria were: clinical infection in at last three months; tuberculosis; intestinal occlusion; pregnancy. All patients were submitted to thorax X-rays, leukogram, tuberculosis cutaneous test. They were treated with infliximabe $5 \mathrm{mg} / \mathrm{kg}$ each two months. They were divided into three groups according to the time of the diagnosis: 5 years, 6 to 10 and more than 10 years. The results were considered better, worse or unchanged. Results - After the initial treatment, $76 \%$ of the patients achieved a response. At the first dose, the ones with 10 years and with associated abdominal surgery had good results and similar to the ones with less than 5 years with no operations. Conclusion - The treatment with infliximab was effective and tolerable in the managing of symptoms in patients with active Crohn's disease, refractory to the conventional treatment and can be a reasonable approach to avoid the surgical treatment.

HEADINGS - Crohn disease. Intestinal inflamatory disease. Monoclonal therapy.

\section{REFERÊNCIAS}

1. Araujo G, Fonseca M. Cost of patient care in patients with Crohn`s disease in Brazil: public health perspective. Value Health. 2007;10(3):A149.

2. Chen IH, Yang KL, Lee A, Huang HH, Lin PY, Lee TD. Low frequency of HLA-B*2706 in Taiwanese patients with ankylosing spondylitis. Eur J Immunogenet. 2002 Oct;29(5):435-8.

3. Chou CT, Chen JM, Hsu CM, Chen SJ. HLA-B27 and its subtypes in 4 Taiwanese Aborigine tribes: a comparison to Han Chinese patients with ankylosing spondylitis. J Rheumatol. 2003 Feb;30(2):321-5.

4. D'Haens G, Baert F, van Assche G, Caenepeel P, Vergauwe P, Tuynman H, De Vos M, van Deventer S, Stitt L, Donner A, Vermeire S, Van de Mierop FJ, Coche JC, van der Woude J, Ochsenkühn T, van Bodegraven AA, Van Hootegem PP, Lambrecht GL, Mana F, Rutgeerts P, Feagan BG, Hommes D; Belgian Inflammatory Bowel Disease Research Group; North-Holland Gut Club. Early combined immunosuppression or conventional management in patients with newly diagnosed Crohn's disease: an open randomised trial. Lancet. 2008 Feb 23;371(9613):660-7.

5. Han PD, Cohen RD. Managing immunogenic responses to infliximab: treatment implications for patients with Crohn's disease. Drugs. 2004;64(16):1767-77.

6. Hanauer SB, Feagan BG, Lichtenstein GR, Mayer LF, Schreiber S, Colombel JF, Rachmilewitz D, Wolf DC, Olson A, Bao W, Rutgeerts P; ACCENT I Study Group. Maintenance infliximab for Crohn's disease: the ACCENT I randomised trial. Lancet. 2002 May 4;359(9317):1541-9.1

7. Hanauer SB. Rev Gastroenterol Disord 2007 Clinical perspectives in Crohn's disease. Turning traditional treatment strategies on their heads: current evidence for "step-up" versus "top-down". Rev Gastroenterol Disord. 2007;7 Suppl 2:S17-22.

8. Hanauer SB. Crohn's disease: step up or top down therapy. Best Pract Res Clin Gastroenterol. 2003 Feb;17(1):131-7.

9. Herrlinger KR, Borutta A, Meinhardt G, Stange EF, Fellermann K. Fatal staphylococcal sepsis in Crohn's disease after infliximab. Inflamm Bowel Dis. 2004 Sep;10(5):655-6.
10. Lichtenstein GR, Abreu MT, Cohen R, Tremaine W; American Gastroenterological Association. American Gastroenterological Association Institute medical position statement on corticosteroids, immunomodulators, and infliximab in inflammatory bowel disease. Gastroenterology. 2006 Mar;130(3):935-9.

11. Lichtenstein GR, Hanauer SB, Sandborn WJ; Practice Parameters Committee of American College of Gastroenterology. Management of Crohn's disease in adults. Am J Gastroenterol. 2009 Feb;104(2):465-83.

12. Lichtenstein GR, Yan S, Bala M, Hanauer S. Remission in patients with Crohn's disease is associated with improvement in employment and quality of life and a decrease in hospitalizations and surgeries. Am J Gastroenterol. 2004 Jan;99(1):91-6.

13. Noguchi M, Hiwatashi N, Liu Z, Toyota T. Secretion imbalance between tumour necrosis factor and its inhibitor in inflammatory bowel disease. Gut. 1998 Aug;43(2):203-9.

14. Salborn WJ, Hanauer SB. AGA Perspectives. American Gastroenterological Association June/July 2007.

15. Sandborn WJ. New concepts in anti-tumor necrosis factor therapy for inflammatory bowel disease. Rev Gastroenterol Disord. 2005 Winter;5(1):10-8.

16. Sands BE, Anderson FH, Bernstein CN, Chey WY, Feagan BG, Fedorak RN, Kamm MA, Korzenik JR, Lashner BA, Onken JE, Rachmilewitz D, Rutgeerts P, Wild G, Wolf DC, Marsters PA, Travers SB, Blank MA, van Deventer SJ. Infliximab maintenance therapy for fistulizing Crohn's disease. N Engl J Med. 2004 Feb 26;350(9):876-85.

17. Targan SR. Biology of inflammation in Crohn's disease: mechanisms of action of anti-TNF-a therapy. Can J Gastroenterol. 2000 Sep;14 Suppl C:13C-16C.

18. van Dullemen HM, van Deventer SJ, Hommes DW, Bijl HA, Jansen J, Tytgat GN, Woody J. Treatment of Crohn's disease with anti-tumor necrosis factor chimeric monoclonal antibody (cA2). Gastroenterology. 1995 Jul;109(1):12935 .

Fonte de financiamento: não há Conflito de interesse: não há Recebido para publicação: 18/01/2009 Aceito para publicação: 02/04/2009 